\title{
ON THE INFLUENCE OF HEAT TRANSPORT ON LOW- FREQUENCY PARAMAGNETIC SPIN-LAT TICE RELAXATION EXPERIMENTS
}

\author{
J. FLOKSTRA, G. J. GERRITSMA, G. A. HARTEMINK \\ and \\ L. C. VAN DER MAREL \\ Technische Hogeschool Twente, Afdeling der Technische Natuurkunde \\ Enschede, The Netherlands
}

Received 7 June 1974

\section{Synopsis}

In low-frequency relaxation experiments on paramagnetic crystals, placed in liquid helium, often dispersion- and absorption curves are found, strongly deviating from those following from the thermodynamic theory of Casimir and Du Pré. For the relaxation time $\tau_{\text {abs }}$, related to the maximum in the absorption curve, a temperature dependence $\tau_{\text {abs }} \propto T^{-\alpha}$ with $1<\alpha<6$ has been found frequently, accompanied sometimes with a jump at the helium-transition temperature $T_{\lambda}$. We computed, starting from a model including the heat conduction of lattice and helium bath and a heat resistance from crystal to bath, dispersion- and absorption curves and found that, assuming the direct process for the spin-lattice relaxation, $\tau_{\mathrm{abs}}(T)$ (related to the maximum of the calculated absorption curve) can be described roughly as mentioned above with a jump at $T_{\lambda}$.

1. Introduction. In the simple thermodynamic theory of Casimir and Du Pré ${ }^{1}$ ) on paramagnetic relaxation in low-frequency alternating fields it is supposed that the magnetic sample is composed of the system of magnetic moments, usually called the spin system, and the lattice. Other assumptions are that the energy transport between these separate thermodynamic systems is proportional to the temperature difference and that the heat conduction in the crystal is very good.

However, relaxation measurements on several paramagnetic crystals, placed in liquid helium, are not in agreement with this thermody namic theory $\left.{ }^{2-6}\right)$. At temperatures above the transition point of helium, $T_{\lambda}$, broadened absorption curves and flattened dispersion curves have been found. For $T<T_{\lambda}$ there is a better agreement with the formulae of Casimir and Du Pré. The deviations from the ideal behaviour are some- 
times expressed in a deviation parameter which, as a function of temperature, has a discontinuity at $T_{\lambda}^{2}$ ). For the temperature dependence of the relaxation parameter $\tau_{\mathrm{abs}}$, defined as the reciprocal value of the frequency $(\omega)$ where the absorption curve has a maximum, often powers of $T$ between -1 and -6 are found. At $T_{\lambda}$ some authors report a discontinuity in the temperature dependence of $\tau_{\text {abs }}$; just above $T_{\lambda}$ this relaxation parameter has a larger value than just below $\mathrm{it}^{3-6}$ ). Tokunaga et al. ${ }^{6}$ ) even found for $T<T_{\lambda}$ another temperature dependence as for $T>T_{\lambda}$.

Several trials have been made to explain the discrepancy between theory and experiment. In early attempts a continuous distribution of relaxation times was introduced ${ }^{7}$ ) and later on agreement was sought by means of a summation of a finite number of dispersion and absorption curves $^{8}$ ). These trials should be considered only as a formal description of the experimental results and up till now they gave no contribution to the explanation of the results, deviating from the simple thermodynamic theory.

In the calculation of Eisenstein ${ }^{9}$ ) allowance is made for the finite heat conduction within the crystal. This calculation was applied to experiments of Van den Broek et al. ${ }^{2}$ ) but here too there was no satisfactory agreement. By taking into account the heat conduction of both the crystal and its surroundings Valkering and one of the present authors (vdM) ${ }^{10}$ ) got a better agreement with some experimental curves.

In this paper we start from an expression for the differential susceptibility as given in ref.10, but in a less complicated presentation. It appears in the first place that experimental and calculated dispersion- and absorption curves can be brought to each other in a satisfactory way. It is demonstrated that the shape of the crystals has some influence on the curves too. In the second place it is found that, supposing the spin-lattice relaxation time $\tau_{\mathrm{s}}$ proportional to $T^{-1}$, the temperature dependence of $\tau_{\text {abs }}$, derived from calculated absorption curves, agrees rather well with many experimental results. At $T_{\lambda}$ we find a discontinuity in the calculated $\tau_{\text {abs }} \nu s . T$ curve.

2. Theory. In this section we present the formulae for the reduced mean susceptibility $\chi$ [see (2.10) of this section] of single crystals with the shape of a sphere, a cylinder and a plate. For a detailed derivation of a general expression for $\chi$ we refer to Valkering et al. ${ }^{10}$ ). Here we only restrict ourselves to the presentation of some essential formulae and equations.

For a paramagnetic crystal in a constant magnetic field $\boldsymbol{H}_{\mathrm{c}}$ with parallel to it a small alternating field $h_{0} \exp i \omega t$ with frequency $\omega$ we suppose: 


$$
\left.\begin{array}{l}
M=M_{\mathrm{c}}+m_{0} \operatorname{expi\omega t} \\
T_{\mathrm{s}}=T_{\mathrm{c}}+\theta_{\mathrm{s}} \operatorname{expi\omega t} \\
T_{\mathrm{L}}=T_{\mathrm{c}}+\theta_{\mathrm{L}} \operatorname{expi\omega t} \\
T_{\mathrm{g}}=T_{\mathrm{c}}+\theta_{\mathrm{g}} \operatorname{expi\omega t}
\end{array}\right]
$$

$\boldsymbol{M}_{\mathrm{c}}$ is the constant part of the magnetization $\boldsymbol{M}$ and $\boldsymbol{m}_{\mathbf{0}}$ the complex amplitude of the alternating part; $T_{\mathrm{c}}$ is the temperature of the bath far from the crystal; $T_{\mathrm{s}}$ is the temperature of the spin system (specific heat at constant field $C_{\mathrm{H}}$ and at constant magnetization $C_{\mathrm{M}}$ ), $T_{\mathrm{L}}$ of the lattice (specific heat $C_{\mathrm{L}}$, coefficient of heat conduction $\lambda_{\mathrm{L}}$ ) and $T_{\mathrm{g}}$ of the helium bath (specific heat $C_{\mathrm{g}}$, coefficient of heat conduction $\lambda_{\mathrm{g}}$ ); $\theta_{\mathrm{s}}, \theta_{\mathrm{L}}$ and $\theta_{\mathrm{g}}$ are complex temperature amplitudes.

The equations describing the interaction between spin system and magnetic field are well known ${ }^{1}$ ) and will not be given here.

The equations describing the heat transport are:

1) between spin system and lattice (heat-transfer coefficient $\alpha$ )

$$
\frac{\mathrm{d} Q_{\mathrm{s}}}{\mathrm{d} t}=-\alpha\left(T_{\mathrm{s}}-T_{\mathrm{L}}\right)
$$

2) in the lattice

$$
\frac{\partial}{\partial t}\left(C_{\mathrm{L}} T_{\mathrm{L}}\right)=\lambda_{\mathrm{L}} \nabla^{2} T_{\mathrm{L}}+\alpha\left(T_{\mathrm{s}}-T_{\mathrm{L}}\right)
$$

3) in the helium bath

$$
\frac{\partial}{\partial t}\left(C_{\mathrm{g}} T_{\mathrm{g}}\right)=\lambda_{\mathrm{g}} \nabla^{2} T_{\mathrm{g}}
$$

At the interface of the crystal and the bath a heat resistance $R_{\mathrm{K}}$ causes a temperature jump. The boundary conditions are:

$$
\frac{1}{R_{\mathrm{K}}}\left(T_{\mathrm{g}}-T_{\mathrm{L}}\right)_{\text {interf. }}=\left(\lambda_{\mathrm{L}} \nabla T_{\mathrm{L}}\right)_{\text {interf. }}=\left(\lambda_{\mathrm{g}} \nabla T_{\mathrm{g}}\right)_{\text {interf. }}
$$

Substituting (2.1) into these equations and eliminating $T_{\mathrm{s}}$ we find the equations for $\theta_{\mathrm{L}}$ and $\theta_{\mathrm{g}}$ : 


$$
\begin{aligned}
& \nabla^{2} \theta_{\mathrm{L}}-L(\omega) \theta_{\mathrm{L}}-M(\omega)=0, \\
& \nabla^{2} \theta_{\mathrm{g}}-\mathrm{i}\left(\omega C_{\mathrm{g}} / \lambda_{\mathrm{g}}\right) \theta_{\mathrm{g}}=0,
\end{aligned}
$$

with

$$
\begin{aligned}
& M(\omega)=-\frac{\alpha}{\lambda_{\mathrm{L}}} \frac{F \mathrm{i} \omega \tau_{\mathrm{s}}}{1+\mathrm{i} \omega \tau_{\mathrm{s}}} h_{0}\left(\frac{\partial T_{\mathrm{s}}}{\partial H}\right)_{M_{\mathrm{c}}}, \\
& L(\omega)=\frac{\alpha}{\lambda_{\mathrm{L}}}\left(1+\mathrm{i} \omega \tau_{\mathrm{s}} \frac{C_{\mathrm{L}}}{C_{\mathrm{H}}}-\frac{1}{1+\mathrm{i} \omega \tau_{\mathrm{s}}}\right), \\
& F=\left(C_{\mathrm{H}}-C_{\mathrm{M}}\right) / C_{\mathrm{H}}, \\
& \tau_{\mathrm{s}}=C_{\mathrm{H}} / \alpha .
\end{aligned}
$$

The reduced differential mean susceptibility will be defined in the usual way as

$$
\chi=\frac{1}{V} \int_{V} \frac{m_{0} / h_{0}}{(\partial M / \partial H)_{T_{c}}} \mathrm{~d} \tau
$$

with $V$ the volume of the crystal and $\mathrm{d} \tau$ a volume element. Following Valkering et $a l .{ }^{10}$ ) we arrive at

$$
\chi=1-F \frac{i \omega \tau_{\mathrm{s}}}{1+\mathrm{i} \omega \tau_{\mathrm{s}}}+F \frac{\mathrm{i} \omega \tau_{\mathrm{s}}}{\left(1+\mathrm{i} \omega \tau_{\mathrm{s}}\right)^{2}} \frac{\alpha / \lambda_{\mathrm{L}}}{M(\omega)} \frac{1}{V} \int_{V} \theta_{\mathrm{L}} \mathrm{d} \tau .
$$

The problem has now been reduced to the solution of the equations (2.6) and (2.7) taking into account that $\theta_{\mathrm{L}}$ has a finite value, that $\theta_{\mathrm{g}}$ approaches to zero at a large distance from the crystal and the two boundary conditions (2.5).

The solution will be presented for spherical crystals, radius $r_{0}$ (section 2.1 ), for cylindrical crystals, radius $r_{0}$, neglecting the influence of the flat boundary planes (section 2.2) and for crystals in the shape of thin plates with thickness $2 r_{0}$ also neglecting the edge effects (section 2.3).

2.1. Spherical crystals. Solutions for (2.6) and (2.7) are respectively given by ${ }^{11}$ ):

$$
\theta_{\mathrm{L}}=A \frac{\sinh (r \sqrt{L)}}{r \sqrt{L}}-\frac{M}{L}
$$




$$
\theta_{\mathrm{g}}=B\left(\mathrm{e}^{\beta r} / \beta r\right)
$$

with

$$
\beta=-(1+\mathrm{i})\left(\omega C_{\mathrm{g}} / 2 \lambda_{\mathrm{g}}\right)^{\frac{1}{2}}
$$

$A$ and $B$ can be calculated with (2.5). For $\theta_{\mathbf{L}}$ is found:

$$
\theta_{\mathrm{L}}=\frac{M}{L}\left[\frac{\sinh (r \sqrt{L})}{N r \sqrt{L}}-1\right]
$$

with

$$
\begin{aligned}
N= & R_{\mathrm{K}} \lambda_{\mathrm{L}} \sqrt{L} \frac{r_{0} \sqrt{L} \cosh \left(r_{0} \sqrt{L}\right)-\sinh \left(r_{0} \sqrt{L}\right)}{r_{0}{ }^{2} L}+\frac{\sinh \left(r_{0} \sqrt{L}\right)}{r_{0} \sqrt{L}} \\
& -\frac{\lambda_{\mathrm{L}}}{\lambda_{\mathrm{g}}} \frac{r_{0} \sqrt{L}}{r_{0} \beta-1} \frac{r_{0} \sqrt{L} \cosh \left(r_{0} \sqrt{L}\right)-\sinh \left(r_{0} \sqrt{L}\right)}{r_{0}{ }^{2} L} .
\end{aligned}
$$

Substitution of (2.15) into (2.11) gives:

$$
\begin{aligned}
\chi= & 1-F \frac{i \omega \tau_{\mathrm{s}}}{1+i \omega \tau_{\mathrm{s}}}+F \frac{i \omega \tau_{\mathrm{s}}}{\left(1+i \omega \tau_{\mathrm{s}}\right)^{2}} \frac{\alpha / \lambda_{\mathrm{L}}}{L} \\
& \times\left[-1+\frac{3}{r_{0}^{2} L}\left\{\frac{R_{\mathrm{K}} \lambda_{\mathrm{L}}}{r_{0}}-\frac{\lambda_{\mathrm{L}}}{\lambda_{\mathrm{g}}} \frac{1}{r_{0} \beta-1}\right.\right. \\
& \left.\left.+\frac{1}{-1+r_{0} \sqrt{L} \operatorname{coth}\left(r_{0} \sqrt{L)}\right.}\right\}^{-1}\right] .
\end{aligned}
$$

2.2. Cylindrical crystals. Solutions for $\theta_{\mathbf{L}}$ and $\theta_{\mathbf{g}}$ are respectively ${ }^{11}$ ):

$$
\begin{aligned}
& \theta_{\mathrm{L}}=A I_{0}(r \sqrt{L)}-M / L, \\
& \theta_{\mathrm{g}}=B\left(\mathrm{ker}_{\mathrm{o}} \gamma r+\mathrm{ikei}_{\mathrm{o}} \gamma r\right),
\end{aligned}
$$

$I_{0}$ is a modified Bessel function, ker and kei are Kelvin functions and $\gamma=\left(\omega C_{\mathrm{g}} / \lambda_{\mathrm{g}}\right)^{\frac{1}{2}}$. Taking into account (2.5) one finds for $\theta_{\mathrm{L}}$ :

$$
\theta_{\mathrm{L}}=\frac{M}{L}\left[\frac{I_{0}(r \sqrt{L})}{N}-1\right]
$$


with

$$
\begin{aligned}
& N=R_{\mathrm{K}} \lambda_{\mathrm{L}} \sqrt{L} I_{1}\left(r_{0} \sqrt{L)}+I_{0}\left(r_{0} \sqrt{L}\right)-\frac{\lambda_{\mathrm{L}}}{\lambda_{\mathrm{g}}} \frac{\sqrt{L}}{\gamma} I_{1}\left(r_{0} \sqrt{L}\right)\right. \\
& \quad \times\left[\frac{\mathrm{ker}_{0} \gamma r_{0}+\mathrm{ikei}_{0} \gamma r_{0}}{\mathrm{ker}_{0}{ }^{\prime} \gamma r_{0}+\mathrm{ikei}_{0}{ }^{\prime} \gamma r_{0}}\right] \\
& \left\{\operatorname{ker}_{0}{ }^{\prime} \gamma r_{0}=\frac{\mathrm{d}}{\mathrm{d}\left(\gamma r_{0}\right)}\left(\operatorname{ker}_{0} \gamma r_{0}\right)\right\} .
\end{aligned}
$$

For the mean reduced susceptibility is found:

$$
\begin{aligned}
\chi= & 1-F \frac{\mathrm{i} \omega \tau_{\mathrm{s}}}{1+\mathrm{i} \omega \tau_{\mathrm{s}}}+F \frac{\mathrm{i} \omega \tau_{\mathrm{s}}}{\left(1+\mathrm{i} \omega \tau_{\mathrm{s}}\right)^{2}} \frac{\alpha / \lambda_{\mathrm{L}}}{L} \\
& \times\left[-1+\frac{2}{r_{0}^{2} L}\left\{\frac{R_{\mathrm{K}} \lambda_{\mathrm{L}}}{r_{0}}-\frac{\lambda_{\mathrm{L}}}{\lambda_{\mathrm{g}}} \frac{1}{\gamma r_{0}} \frac{\mathrm{ker}_{0} \gamma r_{0}+\mathrm{ikei}_{0} \gamma r_{0}}{\mathrm{ker}_{0}{ }^{\prime} \gamma r_{0}+\mathrm{ikei}_{0}^{\prime} \gamma r_{0}}\right.\right. \\
& \left.\left.+\frac{1}{r_{0} \sqrt{L}} \frac{I_{0}\left(r_{0} \sqrt{L}\right)}{I_{1}\left(r_{0} \sqrt{L}\right)}\right\}^{-1}\right] .
\end{aligned}
$$

2.3. Crystals in the shape of thin plates, thickness $2 r_{0}$. If $x$ is the distance to the symmetry plane, which is parallel to the two flat boundary planes, the solutions for $\theta_{\mathrm{L}}$ and $\theta_{\mathrm{g}}$ are $^{11}$ ):

$$
\begin{aligned}
& \theta_{\mathrm{L}}=A \cosh (x \sqrt{L})-M / L, \\
& \theta_{\mathrm{g}}=B \mathrm{e}^{\beta x} .
\end{aligned}
$$

With (2.5) for $\theta_{\mathrm{L}}$ is found

$$
\theta_{\mathrm{L}}=\frac{M}{L}\left[\frac{\cosh (x \sqrt{L})}{N}-1\right]
$$

with

$$
N=\lambda_{\mathrm{L}} R_{\mathrm{K}} \sqrt{L} \sinh \left(r_{0} \sqrt{L}\right)-\frac{\lambda_{\mathrm{L}} \sqrt{L}}{\lambda_{\mathrm{g}} \beta} \sinh \left(r_{0} \sqrt{L}\right)+\cosh \left(r_{0} \sqrt{L}\right) . \text { (2.25) }
$$

For the reduced mean susceptibility it follows 


$$
\begin{aligned}
\chi= & 1-F \frac{\mathrm{i} \omega \tau_{\mathrm{S}}}{1+\mathrm{i} \omega \tau_{\mathrm{s}}}+F \frac{\mathrm{i} \omega \tau_{\mathrm{s}}}{\left(1+\mathrm{i} \omega \tau_{\mathrm{s}}\right)^{2}} \frac{\alpha / \lambda_{\mathrm{L}}}{L} \\
& \times\left[-1+\frac{1}{r_{0}^{2} L}\left\{\frac{\lambda_{\mathrm{L}} R_{\mathrm{K}}}{r_{0}}-\frac{\lambda_{\mathrm{L}}}{\lambda_{\mathrm{g}}} \frac{1}{r_{0} \beta}+\frac{1}{r_{0} \sqrt{L}} \operatorname{coth}\left(r_{0} \sqrt{L}\right\}^{-1}\right] .\right.
\end{aligned}
$$

The three relations for $\chi:(2.16),(2.21)$ and $(2.26)$ have the same structure. The first two terms give the well-known relations of Casimir and $\mathrm{Du}$ Pré. If only the term -1 between the square brackets plays a role the ideally isolated case is found ${ }^{12}$ ). The second term between the square brackets is a correction on both ideal cases. In the denominator of this term $\lambda_{\mathrm{L}} R_{\mathrm{K}} / r_{0}$ represents the influence of the boundary resistance (which is the Kapitza resistance at $T<T_{\lambda}$ ), the next term the influence of the surroundings and the last term the influence of the heat conduction in the crystal.

\section{The influence of the heat transport on the dispersion- and absorp-} tion curves. Starting from expressions (2.16), (2.21) and (2.26) we calculated a number of dispersion- and absorption curves with the aid of a computer. The values of the dimensionless groups, which occur in these relations and which are specified in fig. 1, may greatly influence the shape of these curves. This has especially been investigated for a spherical single crystal.

In fig. 1 , which is an outline of the model, the used quantities are indicated, $D$ and $R$ denote ratios of specific heats; $P, Q$ and $S$ are ratios of coefficients of heat conduction or heat transfer. In order to simplify the expression for the reduced mean susceptibility the dimensionless

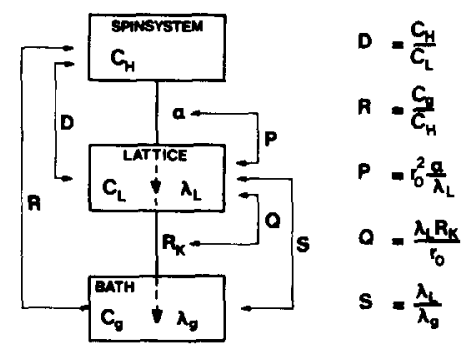

Fig. 1. The model and the dimensionless groups. 
quantities $L^{*}(\omega)\left[=\lambda_{\mathrm{L}} L(\omega) / \alpha\right]$ and $R^{*}\left[=(P R S / 2)^{\frac{1}{2}}\right]$ have been introduced. For expression (2.16) we can now write:

$$
\begin{aligned}
\chi= & 1-F \frac{i \omega \tau_{\mathrm{s}}}{1+\mathrm{i} \omega \tau_{\mathrm{s}}}+F \frac{\mathrm{i} \omega \tau_{\mathrm{s}}}{\left(1+\mathrm{i} \omega \tau_{\mathrm{s}}\right)^{2}} \frac{1}{L^{*}} \\
& \times\left[-1+\frac{3}{P L^{*}}\left\{Q+\frac{S}{(1+\mathrm{i}) R^{*} \sqrt{\omega \tau_{\mathrm{s}}}+1}\right.\right. \\
& \left.\left.+\frac{1}{-1+\sqrt{P L^{*}} \operatorname{coth}\left(\sqrt{P L^{*}}\right)}\right\}^{-1}\right] .
\end{aligned}
$$

Results of the computer calculations on a spherical single crystal with for $F$ the arbitrary value $F=0.5$ will be described now.

3.1. The influence of $P$ and $D$ when $Q=S=0$. The heat resistance $R_{\mathrm{K}}$ has been taken zero and the coefficient of heat conduction $\lambda_{\mathrm{g}}$ infinitely large, so we have to do with the Eisenstein case ${ }^{9}$ ). Indeed it can be shown that (3.1), with $Q=S=0$, is in agreement with the formula of Eisenstein. It appears that for $P<1$ and $D<1$ the deviations from the ideal Casimir and Du Pré curves are small. Dispersion- and absorption curves are presented in fig. 2 for $P=10$ and $P=100$ with different values of $D$. Especially at a large $P$ value the shape and the position of these curves is strongly dependent on $D$. At small values of $D$ the deviations of the ideal behaviour are small and at large values the absorption curves are broadened, mainly at the high-frequency side. It follows from the absorption curve with $P=10$ and $D=100$ that it is not always justified to take the maximum of a nearly ideal absorption curve in the determination of the spin-lattice relaxation time. This situation can occur in systems with large $C_{\mathrm{H}}$ (e.g. at phase transition). A small maximum in the absorption curve appears to be present at $\omega \tau_{\mathrm{s}}=101$, agreeing with the isolated case. The splitting up of the absorption curve at extremely large $P$ in a strongly broadened curve and the ideally isolated curve is obviously demonstrated in fig. 3 .

3.2. The influence of $Q$. For $P$ and $D$ small and $S=0$ expression (3.1) transforms into:

$$
\chi=1-F \frac{i \omega \tau_{\mathrm{s}}}{1+\mathrm{i} \omega \tau_{\mathrm{s}}}+F \frac{\mathrm{i} \omega \tau_{\mathrm{s}}}{\left(1+\mathrm{i} \omega \tau_{\mathrm{s}}\right)^{2}} \frac{1}{L^{*}}\left[-1+\frac{3}{P Q L^{*}+3}\right] .
$$

For large values of $Q$ it can be shown that the dispersion- or absorption 


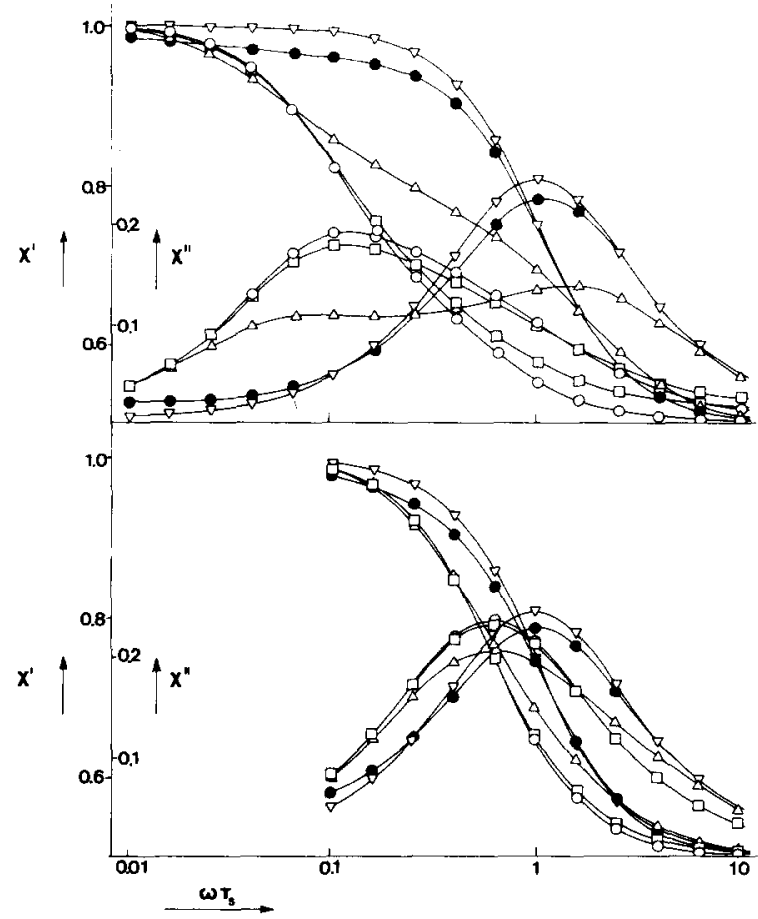

Fig. 2. The dispersion $\left(\chi^{\prime}\right)$ and absorption $\left(\chi^{\prime \prime}\right) v s . \omega \tau_{s}$ (on logarithmic scale) for $P=100$ (upper curves) and $P=10, Q=S=0, F=0.5$ and different $D: \nabla, D=0.01$; - $D=0.1 ; \Delta, D=1 ; \square, D=10 ; \bigcirc, D=100$. There is not much difference between the curves with $D=10$ and $D=100$, which deviate from the ideal behaviour, mainly at the high-frequency side.

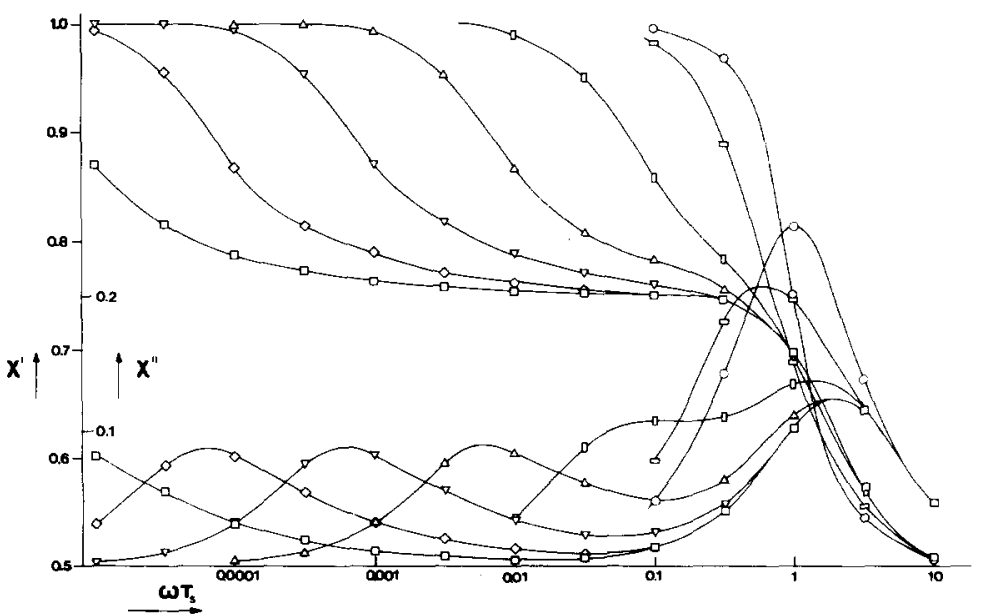

Fig. 3. The dispersion $\left(\chi^{\prime}\right)$ and absorption $\left(\chi^{\prime \prime}\right) v s . \omega \tau_{\mathrm{s}}$ (on logarithmic scale) for $D=1, Q=S=0, F=0.5$ and different $P: 0, P=0.1 ; \square, P=10 ; \square, P=10^{2}$; A. $P=10^{3} ; \nabla, P=10^{4} ; \diamond, P=10^{5} ; \square, P=10^{6}$. 
curve is the sum of two ideal curves, characterized by: $\omega \tau_{\mathrm{s}}=D+1$ (isolated case) and $\omega \tau_{\mathrm{s}}=3 D /[P Q(D+1)]$, i.e. at these values of $\omega \tau_{\mathrm{s}}$ the dispersion curve has a point of inflection and the absorption curve a maximum (fig. 4). In fig. 5 some calculated curves have been given. If the two values of $\omega \tau_{s}$, where the absorption curve has a maximum, are of the same magnitude, it is also allowed to consider this curve as a sum of two ideal absorption curves but it is not possible to connect one of these $\omega \tau_{\mathrm{s}}$ values with a relaxation time of a single relaxation process. Coefficients of heat transfer only give a splitting up into two curves, with unmixed relaxation times, under extreme conditions ${ }^{12,13}$ ).

3.3. The influence of $R$ and $S . P$ and $D$ are supposed to be small and $Q=0$. In figs. 6 and 7 the results have been presented for $R=1$ and 10 , respectively, $S$ varying from 0.1 to 10,000 . At large values of $S$ we get a splitting up in two curves: one at low $\omega \tau_{\mathrm{s}}$ deviating strongly from an ideal curve and one at $\omega \tau_{\mathrm{s}}=D+1$, which is the ideally isolated curve. At small values of $S$ the curves only slightly deviate from those of Casimir and Du Pré. For intermediate values of $S$ flattened dispersion curves and strongly broadened absorption curves are found; the broadening specially being now at the low-frequency side.

At liquid-helium temperatures the value 10 for $S$ seems to be realistic for several crystals on which experiments have been performed. So it is realistic to expect that, as a consequence of the influence of $R$ and $S$, the absorption curves have been broadened at the low-frequency side. Experimental results on crystals, investigated in liquid helium, are in agreement with this expectation ${ }^{2}$ ).

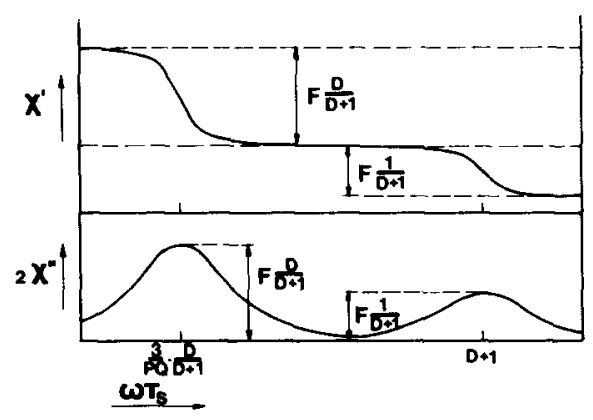

Fig. 4. The $\chi^{\prime}$ and $\chi^{\prime \prime} v s . \omega T_{s}$ curves (on logarithmic scale), when the heat conduction in lattice and bath is good ( $P$ is small, $S=0$ ). The heat transfer coefficients $\alpha$ and $R_{\mathrm{K}}$ bring on two ideal relaxation curves if $(3 / P Q)[D /(D+1)] \ll D+1$. 


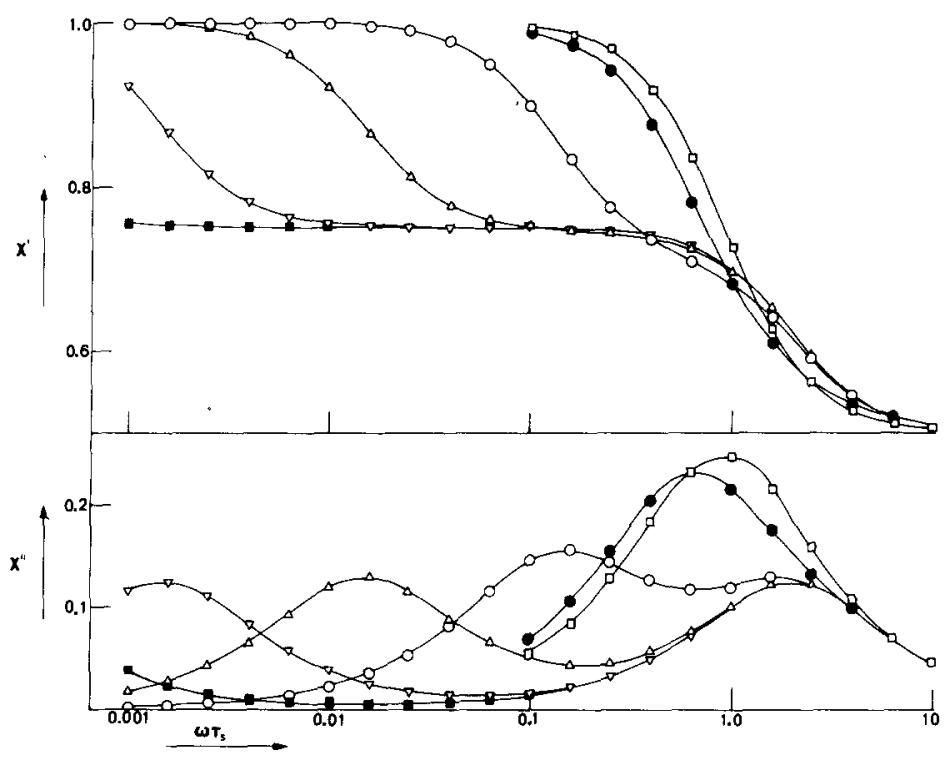

Fig. 5. The dispersion $\left(\chi^{\prime}\right)$ and absorption $\left(\chi^{\prime \prime}\right) v s . \omega \tau_{\mathbf{s}}$ (on logarithmic scale) for $D=1$, $P=1, S=0, F=0.5$ and different $Q: \square, Q=1 ; \bullet, Q=10 ; 0, Q=10^{2} ; \Delta, Q=10^{3} ; \nabla$, $Q=10^{4} ;, Q=10^{5}$. The absorption curve at the low-frequency side reaches the value 0.125 for $Q=10^{4}$ and $Q=10^{5}$ which should be expected for an ideal curve.

4. The temperature dependence of the dispersion- and absorption curves and of $\tau_{a b s}$. In this section we look at the temperature dependence of the calculated dispersion- and absorption curves and of $\tau_{\text {abs }}$. We start from a chosen magnitude of the variable quantities at $T=4.2 \mathrm{~K}$ and calculate their values at lower temperatures from the temperature dependence of these quantities, taken from literature.

1) $F$

2) $D=C_{\mathrm{H}} / C_{\mathbf{L}}$

3) $R=C_{\mathbf{g}} / C_{\mathbf{H}}$

4) $P=r_{0}^{2}\left(\alpha / \lambda_{L}\right)$ independent of temperature.

$C_{\mathrm{H}} \propto T^{-2}$ and $C_{\mathrm{L}} \propto T^{3}$ results in $D \propto T^{-5}$.

$C_{\mathrm{H}} \propto T^{-2}$; the temperature dependence of $C_{\mathrm{g}}$ is given in ref. 14.

We suppose that $\tau_{\mathrm{s}}$ is determined by the direct process, so $\tau_{\mathrm{s}} \propto T^{-1}$ and $\tau_{\mathrm{s}}=C_{\mathrm{H}} / \alpha$ leads to $\alpha \propto T^{-1}$. For $\lambda_{L}$ we suppose: $\lambda_{L} \propto T^{2}$ as is often found for aqueous crystals at $1.0 \mathrm{~K}<T<4.2 \mathrm{~K}^{15}$ ).

5) $Q=\lambda_{\mathrm{L}} R_{\mathrm{K}} / r_{0}$

In our experiments we took zero for this quantity. 


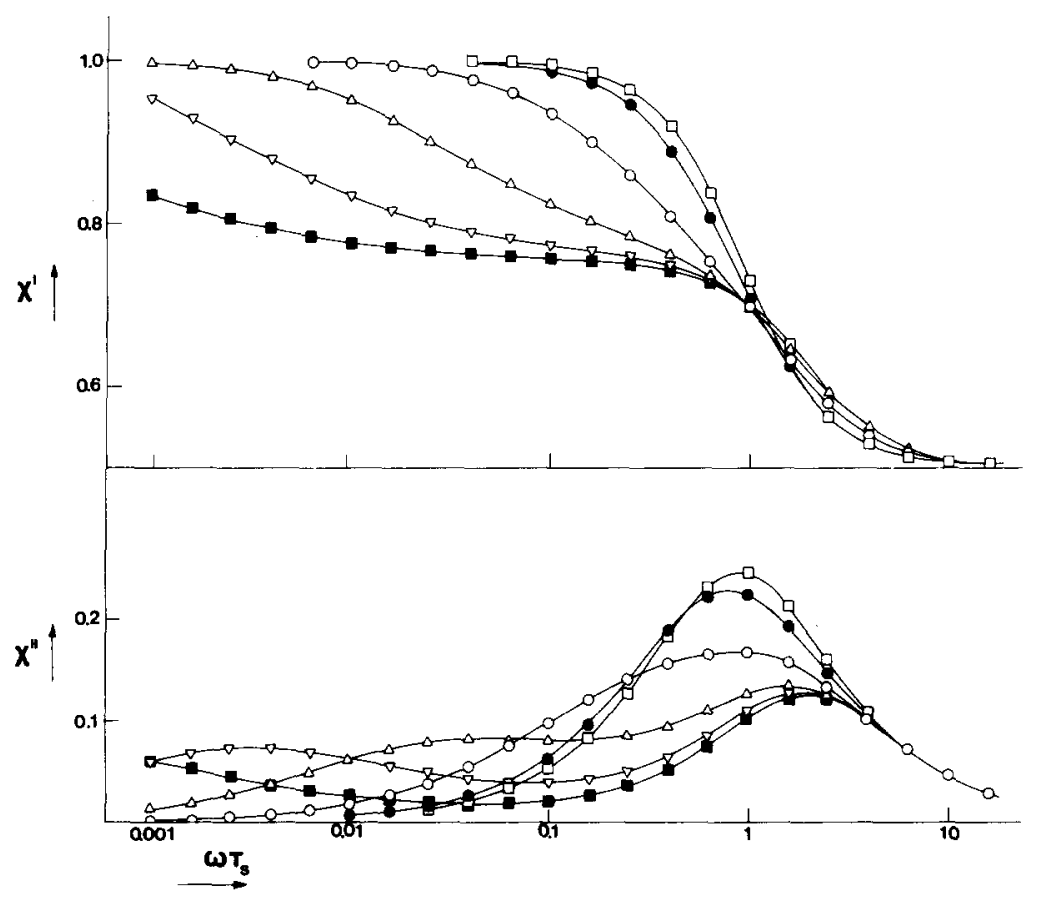

Fig. 6. The dispersion $\left(\chi^{\prime}\right)$ and absorption $\left(\chi^{\prime \prime}\right) v s . \omega \tau_{\mathrm{s}}$ (on logarithmic scale) for $D=1, P=1, Q=0, F=0.5, R=1$ and different $S: \square, S=0.1 ; \bullet, S=1 ; 0, S=10 ; \Delta$, $S=10^{2} ; \nabla, S=10^{3} ; \square, S=10^{4}$. The curves with $S=1$ and $S=10$ are typical experimental ones, with strong deviations mainly at the low-frequency side.

6) $S=\frac{\lambda_{\mathrm{L}}}{\lambda_{\mathrm{g}}}$

$\lambda_{\mathrm{L}} \propto T^{2} ; \lambda_{\mathrm{g}}:$ see ref. 14 . At $T>T_{\lambda} S$ has a finite value and $S=0$ at $T<T_{\lambda}$ because of the extremely good heat-conduction in superfluid helium.

We successively deal with the temperature dependence of the dispersion and absorption curves (section 4.1), the influence of the ratios of coefficients of heat conduction and heat transfer on $\tau_{\mathrm{abs}}$ and on the deviation parameter $d$ to be defined later on (section 4.2) and the influence of the specific heats on $\tau_{\mathrm{abs}}$ and $d$ (section 4.3).

4.1. Dispersion- and absorption curves. In fig. 8 a number of absorption curves, belonging to different temperatures, are presented. The chosen values at $T=4.2 \mathrm{~K}$ of $P, D, S, R, Q$ and $F$ have been given in the figure. The curve of $4.2 \mathrm{~K}$ is already strongly broadened; at decreasing temperature until $2.2 \mathrm{~K}$ this broadening is increasing. The maximum of 


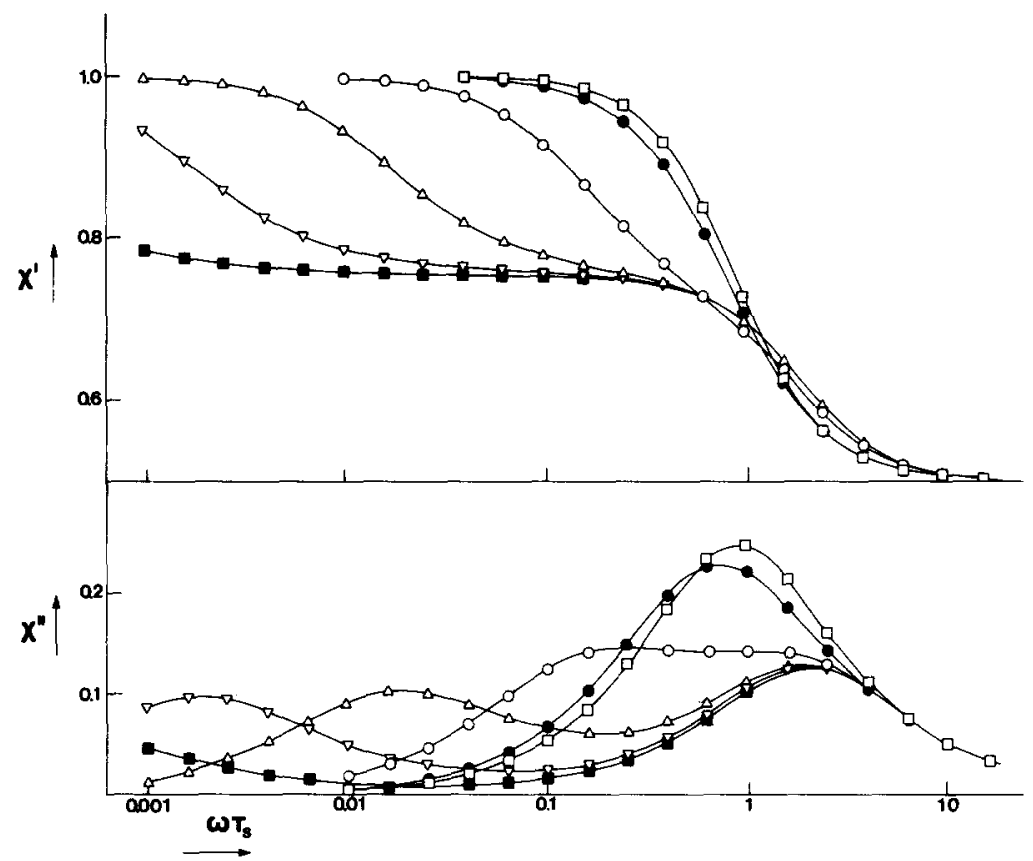

Fig. 7. The dispersion $\left(\chi^{\prime}\right)$ and absorption $\left(\chi^{\prime \prime}\right) v s . \omega \tau_{s}$ (on logarithmic scale) for $D=1$, $P=1, Q=0, F=0.5, R=10$ and different $S: \square, S=0.1 ; \bullet, S=1 ; 0, S=10 ; \Delta$, $S=10^{2} ; \nabla, S=10^{3} ;, S=10^{4}$. The absorption curve at the low-frequency side does not reach the value 0.125 (compare fig. 5).

the absorption is certainly not found at $\omega \tau_{\mathrm{s}}=1$ so the direct process will not be found from it. From $2.2 \mathrm{~K}$ to $2.15 \mathrm{~K}$ the value of $\omega \tau_{\mathrm{s}}$ jumps from 0.20 to 0.54 . The curve of $2.15 \mathrm{~K}$ looks like an ideal Casimir- and Du Pré curve, but the top is not found at $\omega \tau_{\mathrm{s}}=1$. Again an increase of the broadening is found at decreasing temperature. For the dispersion curves, also given in fig. 8 , an analogous behaviour is found.

With the help of the deviation parameter $d=1-2 h / F(h$ is the height of the absorption curve) the deviation from ideal behaviour is described. From the absorption curves of fig. 8 the quantities $\tau_{\mathrm{abs}}(T)$ and $d(T)$ have been derived; the results can be found in fig. 9 and fig. 10 . Notable is the jump of both quantities at $T_{\lambda}$.

4.2. Heat conduction and heat transfer. At a fixed value of $D$ and of $R$ the relative heat-transport quantities $P$ and $S$ have been varied. At $T=4.2 \mathrm{~K}$ we took for $D$ and $R$ the values 1 and 10 respectively which may be in accordance with experimental circumstances of aqueous crystals. In the left-hand part of fig. $9 \tau_{\mathrm{abs}}$ has been plotted as a function of 


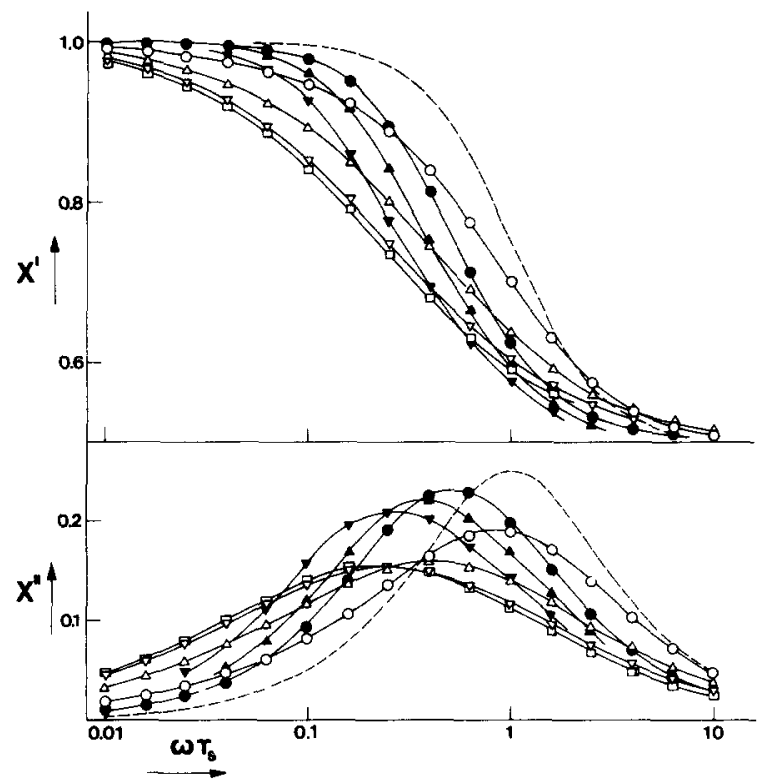

Fig. 8. The dispersion $\left(\chi^{\prime}\right)$ and absorption $\left(\chi^{\prime \prime}\right) v s . \omega \tau_{s}$ (on logarithmic scale) for different temperatures: $O, T=4.2 \mathrm{~K} ; \Delta, T=3.0 \mathrm{~K} ; \nabla, T=2.5 \mathrm{~K} ; \square, T=2.2 \mathrm{~K} ; \bullet$, $T=2.15 \mathrm{~K} ; \Delta, T=1.8 \mathrm{~K} ; \nabla, T=1.5 \mathrm{~K}$. At $4.2 \mathrm{~K}$ the values of the dimensionless groups are: $P=2, D=1, S=10, R=10, Q=0 ; F=0.5$. The dashed curves are the Casimir and Du Pré curves. If there are no deviations all the curves should coincide the ideal curves. Notable is the difference between the curves of $2.2 \mathrm{~K}$ and $2.15 \mathrm{~K}$.

$T$ for $P=0.125$ and 2.0 (at $4.2 \mathrm{~K}$ ) and in the right-hand part of fig. 9 for $P=0.5$ and 8.0. At $T<T_{\lambda}$ the heat conduction in the liquid helium is very good, so $S=0$. For the temperature dependence of $\tau_{\text {abs }}$, derived from the calculated absorption curves, we find:

$$
\tau_{\text {abs }} \propto\left[\begin{array}{lll}
T^{-1.3} & \text { for } & P=0.125 \\
T^{-1.9} & \text { for } & P=0.5 \\
T^{-3.0} & \text { for } & P=2.0 \\
T^{-4.0} & \text { for } & P=8.0 .
\end{array}\right.
$$

So we see a larger temperature dependence for a larger starting value of $P$ at $T=4.2 \mathrm{~K}$. At the $\lambda$-point of helium a jump in $\tau_{\text {abs }}$ is observed; this jump becomes higher when $P$ and $S$ are increasing. The influence of the bath on $\tau_{\text {abs }}$ is clearly demonstrated at temperatures above the $\lambda$-point. The specific heat of liquid helium, which is strongly dependent on temperature just above $T_{\lambda}$, determines the magnitude of $\tau_{\mathrm{abs}}$ in such a way 


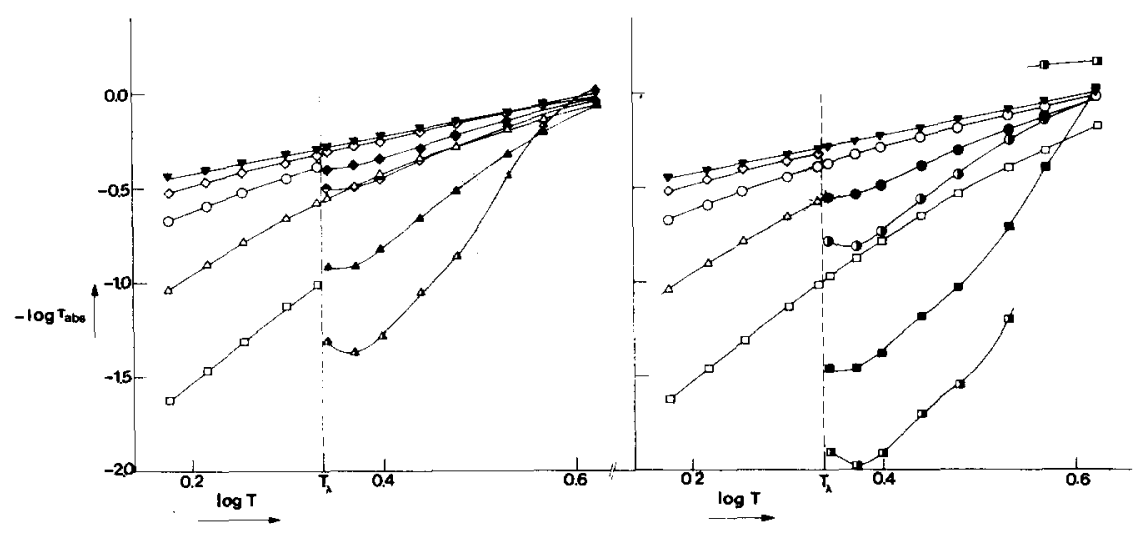

Fig. 9. The influence of $P$ and $S$ on the $\tau_{\text {abs }} v s$. $T$ curve. We computed $\tau_{\text {abs }}(T)$ making use of the direct process for $\tau_{\mathrm{s}}$ given by $\nabla$. The values of $\tau_{\mathrm{s}}(T)$ and $\tau_{\mathrm{abs}}(T)$ are normalized, that means they have been divided by the value of $\tau_{\mathrm{s}}$ at $4.2 \mathrm{~K}$.

At $4.2 \mathrm{~K}: D=1, R=10 ; Q=0, F=0.5$

\begin{tabular}{rcccc}
\hline \multicolumn{1}{c}{$P$} & $P=0.125$ & $P=0.5$ & $P=2.0$ & $P=8.0$ \\
\hline 0 & $\diamond$ & 0 & $\Delta$ & $\square$ \\
10 & $\diamond$ & $\bullet$ & $\Delta$ & $\square$ \\
40 & $\diamond$ & $\bullet$ & $\Delta$ & $\square$ \\
\hline
\end{tabular}

For clarity's sake the curves have been separated into two figures; the left-hand one for $P=0.125$ and $P=2.0$, the right-hand one for $P=0.5$ and $P=8.0$. The curve, indicated by $\theta$, nearly coincides the one indicated by $\Delta$ (for $T>T_{\lambda}$ ).

that a minimum in the $\tau_{\mathrm{abs}} v s . T$ curve is found in some cases at about $2.3 \mathrm{~K}$. In the temperature region from $3.0 \mathrm{~K}$ to $4.2 \mathrm{~K}$ sometimes two tops are observed in the absorption curve: one as a consequence of the isolated lattice and the other one as a consequence of the bad heat conduction in the lattice and the bath.

In fig. 10 the deviation parameter $(d)$ has been given for a number of curves, presented in fig. 9. We remark that: at $T_{\lambda}$ the $d v s$. $T$ curve may have a jump, which magnitude is dependent on $P$ and $S$; for $T>T_{\lambda}$ the deviation parameter $d$ may be very large $(0.5-0.6)$; for $T<T_{\lambda} d$ is larger as $P$ is larger and $d$ is increasing at decreasing temperature.

\subsection{Specific heats. At a fixed value of $P$ and of $S$ the relative} specific-heat quantities $D$ and $R$ have been varied. On account of data from literature we chose $P=0.50$ and $S=10$ (both at $T=4.2 \mathrm{~K}$ ); $Q$ has been taken zero and $D 0.1,1$ and 10 . The results have been presented in fig. 11. For $T<T_{\lambda}$ the magnitude of $D$ has hardly any influence on $\tau_{\mathrm{abs}}$. 


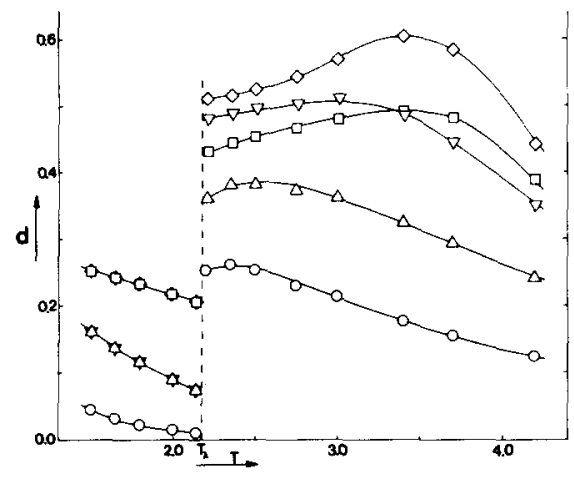

Fig. 10. The deviation parameter $d=1-2 h / F v s$. $T$ for different $P$ and $S$. At $4.2 \mathrm{~K}: D=1, R=10, Q=0, F=0.5$.

\begin{tabular}{clc}
\hline & $P$ & $S$ \\
\hline$\circ$ & 0.50 & 10 \\
$\Delta$ & 2.0 & 10 \\
$\nabla$ & 2.0 & 40 \\
$\square$ & 8.0 & 10 \\
$\diamond$ & 8.0 & 40 \\
\hline
\end{tabular}

This effect we already observed for large values of $D$ in fig. 2. Almost independent of $D$ we find $\tau_{\text {abs }} \propto T^{-1.9}$. The $\tau_{\text {abs }} v s$. $T$ curve has a discontinuity at $T_{\lambda}$ again. The magnitude of the jump is mainly determined by $R$ : the larger $R$, the smaller the jump. A large $R$ means a relative large specific heat of the surrounding helium and so the influence of the properties of heat conduction of the helium on $\tau_{\mathrm{abs}}$ is only small. For $T>T_{\lambda}$ the curves of $R=0.1$ and 0.01 almost coincide; partly this is the case for the curves with $R=1$. Again we see that in a definite temperature region the absorption curve may have two maxima, i.e. there are two values of $\tau_{\text {abs. }}$.

In section 4.2 the specific heat ratios and in section 4.3 the heat-transport ratios are kept constant. Actually a variation of all the quantities should be executed. We are of the opinion that by doing so an incalculable quantity of data might be obtained. Therefore we believe that it is preferable to start from relative quantities, derived from experiment. The specific results, presented in sections 4.2 and 4.3 , will be found undoubtedly. 


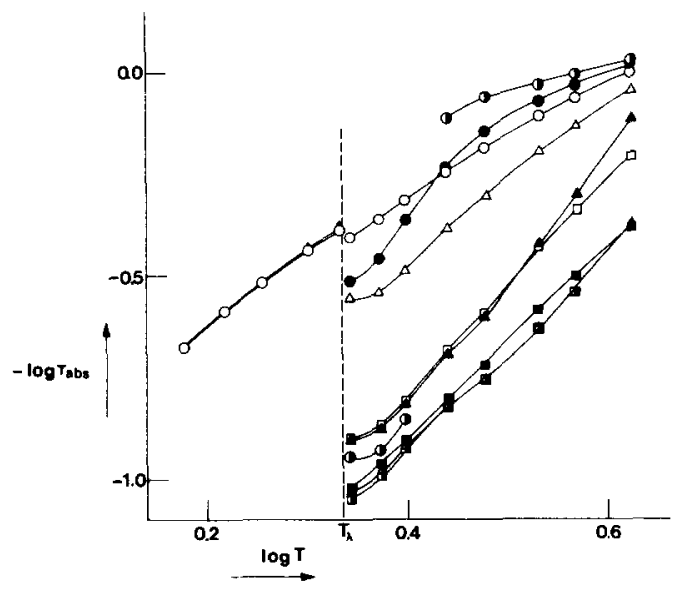

Fig, 11. The influence of $D$ and $R$ on the $\tau_{\text {abs }}$ vs. $T$ curve. The values of $\tau_{\text {abs }}(T)$ are normalized (see caption of fig. 9). At $4.2 \mathrm{~K}: P=0.50, S=10 ; Q=0, F=0.5$

\begin{tabular}{rlrrrrr}
\hline$D$ & \multicolumn{8}{c}{$R$} \\
\hline 0.1 & 1 & $\bullet$ & 10 & $\bullet$ & 100 & 0 \\
1 & 0.1 & $\Delta$ & 1 & $\bullet$ & 10 & $\Delta$ \\
10 & 0.01 & $\star$ & 0.1 & $\bullet$ & 1 & $\square$ \\
\hline
\end{tabular}

For $T<T_{\lambda}$ the three curves with $D=0.1,1$ and 10 respectively nearly coincide.

5. Influence of the crystal shape. The susceptibility for spherical and cylindrical crystals and for crystals in the shape of thin plates has been given by the expressions (2.16), (2.21) and (2.26), respectively. The term between square brackets in these formulae, denoted by $f_{\mathrm{s}}, f_{\mathrm{c}}$ and $f_{\mathrm{p}}$, respectively, is a complex quantity and, for the three cases, not mutually comparable in a simple way. The boundary values of $f$ are 0 (Casimir and Du Pré case) and - 1 (ideally isolated case). If only the Eisenstein term is taken into account for small values of the argument $z=r_{0} \sqrt{ } L$, it is possible to compare the three $f$ 's with each other. We find in this case: $f_{\mathrm{s}}=-\frac{1}{15} z^{2}, f_{\mathrm{c}}=-\frac{1}{8} z^{2}$ and $f_{\mathrm{p}}=-\frac{1}{3} z^{2}$. So for an equal value of $z$ (that means: the same value for $r_{0}$ and the same material) it holds: $\left|f_{\mathrm{p}}\right|>\left|f_{\mathrm{c}}\right|$ $>\left|f_{s}\right|$ and therefore the curves for the plate have the largest deviations from the ideal curves of the Casimir and Du Pré theory. In view of the limited possibilities of heat transport of the plate this could have been expected in advance. In practice experiments are often performed on cylindrical crystals and in order to measure the same effect (about the same number of spins in the crystal) the radius of a cylinder can be taken 
smaller than that of a sphere. Actually it is preferable to do experiments with thin plates or with long cylinders rather than with a sphere. From this consideration it also follows that better results can be expected with powders ${ }^{16}$ ).

In fig. 12 the dispersion- and absorption curves have been presented for the three crystal shapes with $D=1, R=10, P=5, Q=0, S=0$ and $F=0.5$. It can be seen that the plate gives the largest deviations.

In fig. 13 the temperature dependence of $\tau_{\text {abs }}$ has been given, calculated from the starting values $D=1, R=10, P=2, Q=0, S=10$ and $F=0.5$ at $T=4.2 \mathrm{~K}$ and a temperature dependence of the parameters given in the preceding section.

For $T<T_{\lambda}$ we find:

$$
\begin{array}{ll}
\text { sphere } & \tau_{\mathrm{abs}} \propto T^{-3.0}, \\
\text { cylinder } & \tau_{\mathrm{abs}} \propto T^{-3.3}, \\
\text { plate } & \tau_{\mathrm{abs}} \propto T^{-3.9} .
\end{array}
$$

6. Comparison with some experimental results. As already mentioned in section 1 the experimental results of Van den Broek et al. ${ }^{2}$ ) on a spherical sample of $\mathrm{KCr}$ alum can be better described with the formulae of Valkering et al. ${ }^{10}$ ) than with those of Eisenstein. The change in the dispersion and absorption curves at $T=T_{\lambda}$ was explained by assuming that superfluid helium penetrates the pores of the crystal and this should improve the heat transport. In our model we suppose that for $T>T_{\lambda}$ the

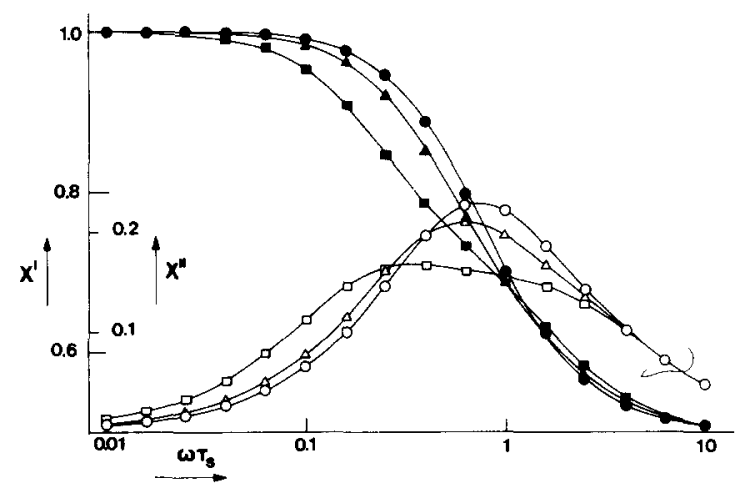

Fig. 12. The dispersion $\left(\chi^{\prime}\right)$ and absorption $\left(\chi^{\prime \prime}\right) v s . \omega \tau_{s}$ (on logarithmic scale) for the three crystal shapes: sphere $(\bullet, O)$, cylinder $(\Delta, \triangle)$ and plate $(\bullet, \square) ; D=1, R=10, P=5$, $Q=0, S=0$ and $F=0.5$. 


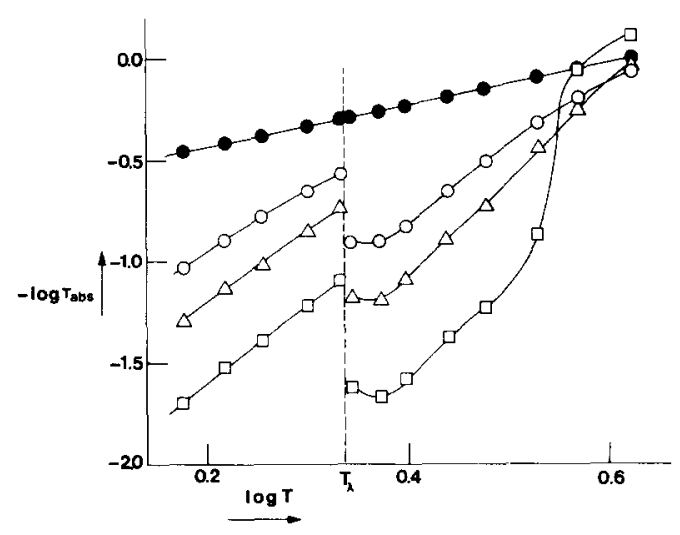

Fig. 13. The influence of the shape of the crystal on the $\tau_{\text {abs }}$ vs. $T$ curve. We computed $\tau_{\text {abs }}(T)$ making use of the direct process for $\tau_{\mathrm{s}}$ given by $\bullet$. The values of $\tau_{\mathrm{s}}(T)$ and $\tau_{\text {abs }}(T)$ are normalized, that means: they have been divided by the value of $\tau_{\mathrm{s}}$ at 4.2 K. Sphere: $O$; cylinder: $\triangle$; plate: $\square . D=1, R=10, P=2, Q=0, S=10$ and $F=0.5$.

relaxation phenomena are greatly determined by the heat properties of the helium and of the crystal while for $T<T_{\lambda}$ only the Eisenstein term remains. This implies that at decreasing temperature the deviations from ideal behaviour will increase again and indeed this is in accordance with experimental results ${ }^{17}$ ).

In this section we present calculated values of $\tau_{\mathrm{abs}}(T)$ of $\mathrm{KCr}$ alum at $H=2250 \mathrm{Oe}$ and we compare these with the measurements of Van den Broek et al. ${ }^{2}$ ). For the determination of $C_{\mathrm{H}}$ and $C_{\mathrm{M}}$ we use: $\mu_{0} C=$ $109 \times 10^{-9} \mathrm{~J} \mathrm{~K} / \mathrm{A}^{2} \mathrm{~m}$ and $b / \mu_{0} C=4.5 \times 10^{9} \mathrm{~A}^{2} / \mathrm{m}^{2}$. The radius $r_{0}$ of the crystal is $8 \times 10^{-3} \mathrm{~m}$. At $T=4.2 \mathrm{~K}$ we take for $\lambda_{\mathrm{L}}=1.8 \mathrm{~J} / \mathrm{Ksm}$ and for $\tau_{\mathrm{s}}=1.5 \times 10^{-3} \mathrm{~s}$; it often appears (figs. 9,11 ) that at $4.2 \mathrm{~K}$ the difference between the calculated $\tau_{\mathrm{abs}}$ and the spin-lattice relaxation time $\tau_{\mathrm{s}}$ generally is small. For $C_{\mathrm{L}}$ we take the results of Kapadnis ${ }^{18}$ ): $C_{\mathrm{L}}=$ $15.07 \times T^{3}\left(\mathrm{~J} / \mathrm{K} \cdot \mathrm{m}^{3}\right)$. The data of liquid helium $\lambda_{\mathrm{g}}$ and $C_{\mathrm{g}}$, we borrow from Wilks ${ }^{14}$ ). The temperature dependence of the used quantities has been given in section 4 . At $T=4.2 \mathrm{~K}$ we calculate: $D=0.20, R=2500$, $S=63.5, P^{\prime}=5.4$ and $F=0.88 ; Q$ is taken zero.

In fig. 14 our calculated values and those of Van den Broek et al. have been presented. The agreement is not unsatisfactory. The calculated values can roughly be described by: $\tau_{\text {abs }} \propto T^{-3.5}$ whereas Van den Broek et al. found: $\tau_{\text {abs }}(\exp ) \propto T^{-3.65}$. The jump at $T_{\lambda}$ is not large in the calculated curve and in the description of the experiments no mention is made of it.

We also applied the model to some other aqueous crystals. So e.g. it is 


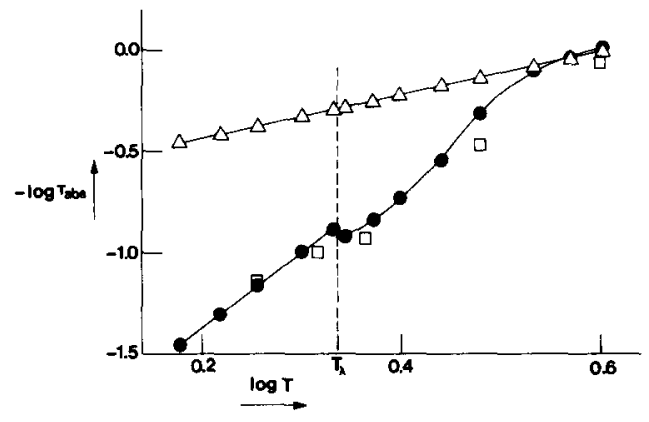

Fig. 14. The $\tau_{\text {abs }}$ vs. $T$ curve for chromium potassium alum at $H=2250 \mathrm{Oe}$. We computed $\tau_{\text {abs }}(T)$ indicated by $\bullet$ making use of the direct process for $\tau_{\mathrm{s}}$ indicated by $\triangle$. The values, measured by Van den Broek et al., are also indicated ( $\square$ ). All the $\tau$ 's are normalized, that means they have been divided by the estimated value of $\tau_{\mathrm{s}}$ at $4.2 \mathrm{~K}$.

possible to explain why the experimental results of two of the present authors $\left.\left(\mathrm{F}, \mathrm{vdM}^{4}\right)\right)$ on cerium magnesium nitrate are in excellent agreement with Casimir and Du Pré's formulae whereas the curves of lanthanum manganese nitrate in fields from about 6 kOe show large deviations. In the last mentioned salt a discontinuity in the $\tau_{\text {abs }} v s$. $T$ curve has been found.

For many crystals the right value for $\lambda_{L}$ is a problem. Bijl ${ }^{15}$ ) found for chromium potassium alum that $\lambda_{L}$ is dependent on the way of cooling the crystal. This implies that relaxation measurements may be not quite reproducible. This indeed we observed in recent experiments on $\mathrm{Cs}_{2} \mathrm{MnCl}_{4} \cdot 2 \mathrm{H}_{2} \mathrm{O}$.

Finally we remark that in relaxation experiments with an exponential recovery curve (e.g., pulse saturation with monitored recovery), probably the influence of the heat conduction in the crystal (especially aqueous crystals) and in the surroundings has also to be taken into account. In this case it will not be possible to describe the recovery curve with one single exponential power.

7. Conclusions. In the preceding sections we demonstrated that the heat conduction in the crystal and in the surrounding helium may influence largely the shape of the dispersion- and absorption curves. On one side we see curves, strongly deviating from the ideal case, on the other the curves may be largely moved up to low frequencies. In case of bad heat conduction the temperature dependence of $\tau_{\mathrm{abs}}$ (for $\omega \tau_{\mathrm{abs}}=1$ the absorption curve has a maximum) is not so much determined by the spinlattice relaxation, but more by the temperature dependent heat-transport quantities. At $T=T_{\lambda}$ we see a jump in the calculated $\tau_{\text {abs }}$ vs. $T$ curve as a 
consequence of properties of the surrounding helium. So it is not necessary to assume a penetration of superfluid helium into the pores of the crystal in order to explain the nearly ideal curves at $T<T_{\lambda}$.

We are convinced of the fact that the model discussed in this paper has its restrictions. So we adopted one spin system and one lattice system. Extension of the model with more systems will undoubtedly be possible. If the crystal is surrounded by helium gas the model should be extended as well. On the crystal there will be a helium film, fluctuating in thickness. The influence of the pressure of the helium gas on the dispersion- and absorption curves is now studied in our group. Indeed we find a strong pressure dependence in recent experiments on $\mathrm{Cs}_{2} \mathrm{MnCl}_{4} \cdot 2 \mathrm{H}_{2} \mathrm{O}$, which has bad heat conduction. In order to avoid the influence of the heat conduction on the relaxation phenomena we hope, with a low pressure of the surrounding helium gas, to acquire real information about the spin-lattice relaxation time.

Acknowledgements. The authors wish to thank Mrs. G. E. van Kan-Kloek for drawing the figures and Miss E. de Boer for typing the manuscript. The cooperation with the university computer centre "Rekencentrum" during the calculations has been gratefully acknowledged.

\section{REFERENCES}

1) Casimir, H. B. G. and Du Pré, F. K., Physica 5 (1938) 507.

2) Van den Broek, J., Van der Marel, L. C. and Gorter, C. J., Physica 27 (1961) 661.

3) Von Klot, R., Phys. kondens. Materie 6 (1967) 290.

4) Flokstra, J., Verheij, W. A., Van der Marel, L. C., Bots, G. J. C. and Van de Klundert, L. J. M., Phys. Letters 40 (1972) 363.

5) Flokstra, J., Gerritsma, G. J., Vermeulen, A. J. W. A. and Botterman, A. C., Phys. Letters 44 (1973) 485.

6) Tokunaga, Y., Ikeda, S., Ito, K. and Haseda, T., J. Phys. Soc. Japan 35 (1973) 1353.

7) Cole, K. S. and Cole, R. H., J. chem. Phys. 9 (1941) 341. Fuoss, R. and Kirkwood, S. G., J. Am. chem. Soc. 63 (1941) 385.

8) Van der Marel, L. C., Verstelle, J. C. and Gorter, C. J., Proc. 8th Int. Conf. Low Temp. Phys., Butterworths (London, 1963) p. 289.

9) Eisenstein, J., Phys. Rev. 84 (1951) 548.

10) Valkering, T. P. and Van der Marel, L. C., Physica 47 (1970) 412.

11) Abramowitz, M. and Segun, I. A., Handbook of Mathematical functions, Dover Publications (New York, 1964).

12) Van der Marel, L. C., Thesis Leiden, 1958. Van der Marel, L. C., Bots, G. J. C. and Blaisse, B. S., Proc. 15th Coll. AMPERE (Grenoble, 1968) p. 480.

13) Sauermann, G. and Schwegler, H., Z. für Physik 209 (1968) 355.

14) Wilks, J., The Properties of Liquid and Solid Helium, Clarendon Press (Oxford, 1967).

15) Bijl, D., Physica 14 (1949) 684. 
16) Roest, J. A., Van Duyneveldt, A. J., Van der Bilt, A. and Gorter, C. J., Physica 64 (1973) 306.

17) Lasheen, M. A., Van den Broek, J. and Gorter, C. J., Physica 34 (1958) 1061.

18) Kapadnis, D. G., Physica 22 (1956) 159. 